\section{Mutual Alignment of Block Copolymer-Magnetic Nanoparticle Composites in a Magnetic Field}

\section{Matthew R. Hammond, ${ }^{*}{ }^{\dagger}$ Hervé Dietsch, Olivier Pravaz, and Peter Schurtenberger*,}

Adolphe Merkle Institute, University of Fribourg. Rte. de l'ancienne Papeterie CP 209, 1723 Marly 1, Switzerland

Present address: National Institute of Standards and

Technology, 100 Bureau Drive, MS 8542, Gaithersburg,

MD 20899. "Present address: Division of Physical Chemistry,

Center for Chemistry and Chemical Engineering,

Lund University, PO Box 124, SE-221 00 Lund, Sweden.

For the past $\sim 30$ years, polymer nanocomposites $(\mathrm{PNCs})^{1}$ and block copolymers $(\mathrm{BCPs})^{2}$ have represented two extremely active areas of research within the polymer science community. It was inevitable, then, that the two topics should overlap; block copolymer/nanoparticle composites represent a relatively new and exciting field in materials research. A large portion of such work has, to date, sought to employ the well-ordered microphaseseparated structures available in block copolymers as scaffolds to direct the spatial arrangement of the nanofiller. ${ }^{3,4}$ Indeed, effective control over the spatial location and/or orientation of the nanofiller particles in PNCs offers great possibilities for dramatically improved composite properties. ${ }^{1}$ In the block copolymer field, great strides have been made in developing techniques to globally orient the BCP microdomains using external fields ${ }^{5}$ (e.g., shear ${ }^{6}$ or electric fields $\left.{ }^{7}\right)$ or surfaces, ${ }^{8-11}$ even further enhancing the possibility for the creation of nanocomposites with precisely defined morphology.

In this Communication, we present a general method for creation of aligned block copolymer/nanoparticle (BCP/NP) composites, although via something of an inverse approach: an external (magnetic) field is used to define the spatial orientation of rodshaped magnetic NPs, which then serve as structure-directing agents for neighboring BCP domains. Specifically, the oriented NPs, which are incorporated at a concentration of only a few percent, present templating surfaces for the alignment of cylindrical block copolymer nanodomains. In a sense, our approach is an extrapolation of the graphoepitaxy technique, which can be used to template BCP cylinder orientation in thin films, ${ }^{10,11}$ to thicker films, and eventually into the bulk. The ability of rod-shaped NPs to nucleate coaxially oriented BCP cylinders has recently been established. ${ }^{12,13}$ There has also been a fair amount of work using magnetic fields to orient block copolymers, although it is necessary to use copolymers which contain moieties or semicrystalline domains with appreciable magnetic susceptibility. ${ }^{5,14-17}$ In the absence of such a condition, we introduced the rod-shaped nanoparticles, which are capable of being magnetically aligned. Using this kind of scheme to induce reorientation in lyotropic liquid crystal/nanomagnet composites has also been demonstrated. ${ }^{18,19}$

The composite under study consisted of a polystyrene-blockpoly(2-vinylpyridine) (PS-P2VP) diblock copolymer, which forms cylindrical microdomains of P2VP in a PS matrix, ${ }^{20}$ and spindletype hematite $\left(\alpha-\mathrm{Fe}_{2} \mathrm{O}_{3}\right)$ particles, which were synthesized by the forced hydrolysis of $\mathrm{Fe}\left(\mathrm{ClO}_{4}\right)_{3}$, according to procedures previously

*Corresponding authors. E-mail: matthew.hammond@mailaps.org (M.R.H.), peter.schurtenberger@fkem1.lu.se (P.S.).
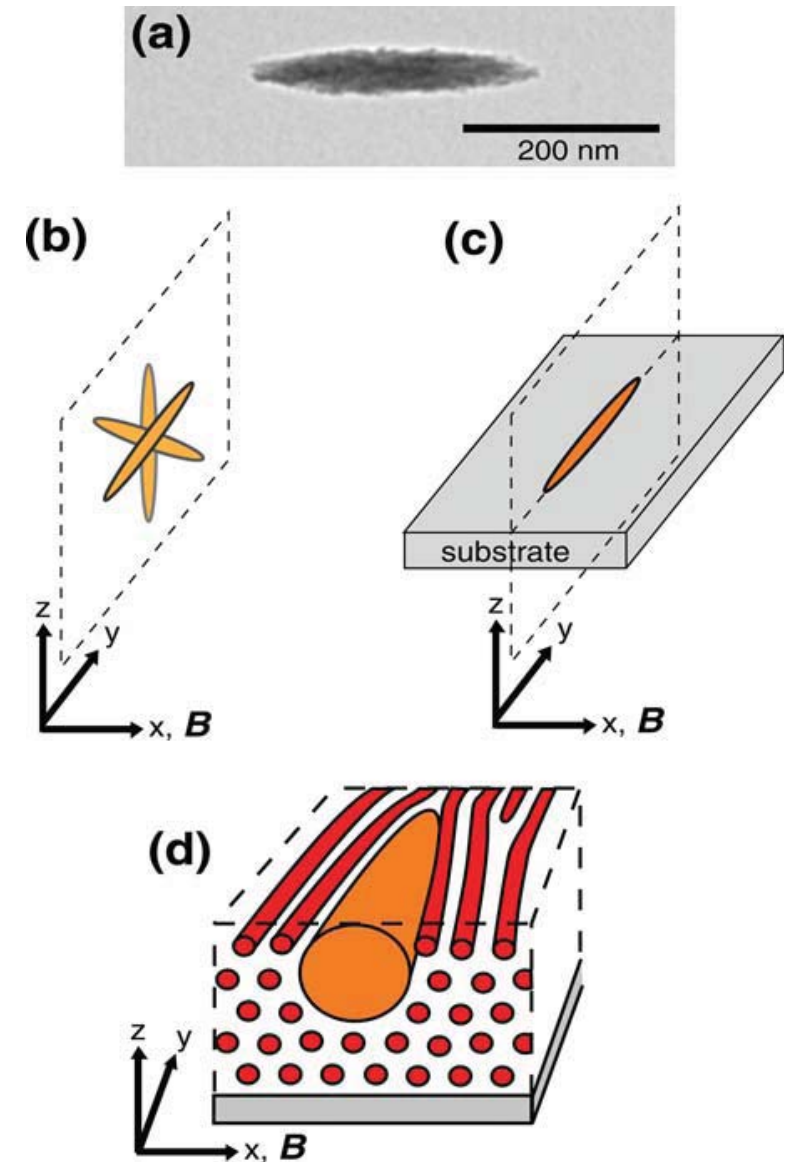

Figure 1. (a) TEM image of a typical needle-shaped hematite particle. (b) The particles orient in the plane perpendicular to the applied magnetic field, $\boldsymbol{B}$. (c) Confining the particles to lie within the block copolymer film parallel to the substrate, and under the influence of $\boldsymbol{B}$, should result in a unique particle orientation. (d) The preferred BCP cylinder orientation is parallel to both the substrate and the long axis of neighboring hematite nanoparticles.

described by Ocaña et al. ${ }^{21}$ The particles, an example of which is shown in the transmission electron microscopy (TEM) image in Figure 1a, had an average diameter of $55 \pm 6 \mathrm{~nm}$ and an average long axis length of $330 \pm 38 \mathrm{~nm}$ (see the Supporting Information, Figure S1). Such hematite particles are weakly ferromagnetic and have recently been shown to orient with their major axes perpendicular to an applied magnetic field, $\boldsymbol{B},{ }^{22}$ as drawn in Figure $1 \mathrm{~b}(\boldsymbol{B}$ applied in the $x$-direction). To overcome this problem and introduce a unique particle orientation, the composites were cast as films of thickness $\sim 500 \mathrm{~nm}$; by confining the particles to lie in the film plane and perpendicular to the applied magnetic field, the particles should orient preferentially in the $y$-direction diagrammed in Figure 1c. Films were produced by casting a THF solution of BCP (1 wt \%) and NP $(0.05 \mathrm{wt} \%)$ onto either carbon-coated epoxy or mica substrates, followed by slow solvent evaporation. For samples subjected to the magnetic field, the substrate was placed between two permanent magnets ${ }^{23}$ and the solution casting procedure followed. To allow the $\mathrm{BCP}$ microstructure to evolve toward equilibrium, the films were annealed in $\mathrm{CH}_{2} \mathrm{Cl}_{2}$ solvent vapor (a neutral solvent for PS and P2VP) ${ }^{24}$ for 1 day while still under the influence of the magnetic field. This process resulted in the alignment of the BCP cylinders with 

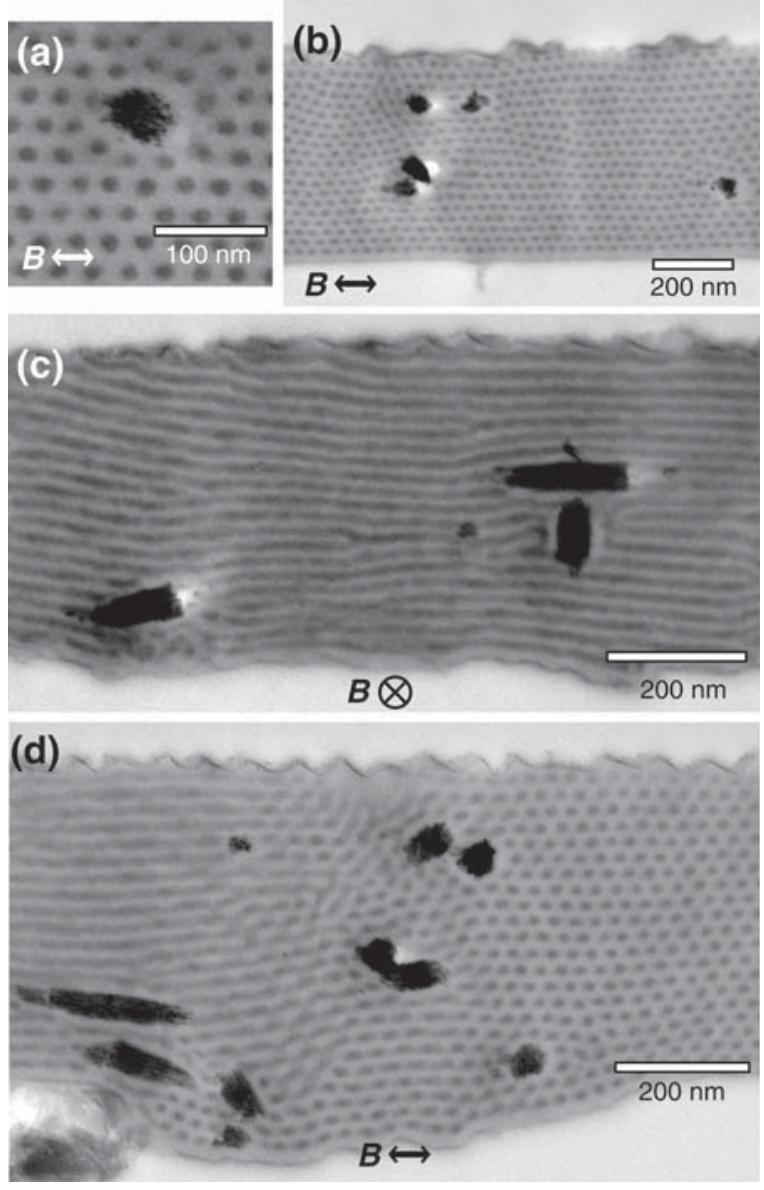

Figure 2. TEM images of a BCP/NP composite film cross-sectioned parallel (a, b, d) and perpendicular (c) to the applied magnetic field, $\boldsymbol{B}$.

their axes predominantly parallel to the long axis of nearby hematite particles, as schematized in Figure 1d.

To elucidate the structure in the nanocomposite films, TEM was performed on film cross sections, with iodine staining of the $\mathrm{P} 2 \mathrm{VP}$ domains performed to allow visualization of the BCP structure (Figure 2). Sectioning was performed both parallel to the applied magnetic field direction (the $x z$ plane as indicated in Figure 1) and perpendicular to $\boldsymbol{B}$ (the $y z$ plane). Figure 2 a shows a single particle (in cross section) embedded in the PS-rich matrix of the BCP. P2VP is known to strongly wet many oxide, ${ }^{9}$ metal, ${ }^{25}$ and semiconductor ${ }^{26}$ surfaces. This is presumably the case with the hematite particles, as well, leading to the formation of a PS-P2VP brush layer that compatibilizes the particles within the PS matrix of the BCP. ${ }^{27}$ Since the remanent magnetic moment of the particles is rather low, ${ }^{22}$ the steric repulsion between particles afforded by this brush layer is sufficient to prevent particle aggregation in solution and in the composites. Images from cross sections cut parallel to $\boldsymbol{B}$ typically show both particles and P2VPrich cylinder cores in cross section, as in Figure 2a,b. The cylinders show the usual hexagonal packing, except in proximity to a particle, where lattice distortions are often observable. Given that the particle diameter is much larger than the BCP domain periodicity, this is to be expected. Cross sections cut perpendicular to $\boldsymbol{B}$, as in Figure 2c, typically show particles and cylinders lying with their principal axes lying in the plane of the section. Note that the thickness of the sections is comparable to the particle diameter, so in many instances the particles are not left entirely intact by the microtoming procedure. Again, the fact that the particle size and shape are not perfectly commensurate with (a)
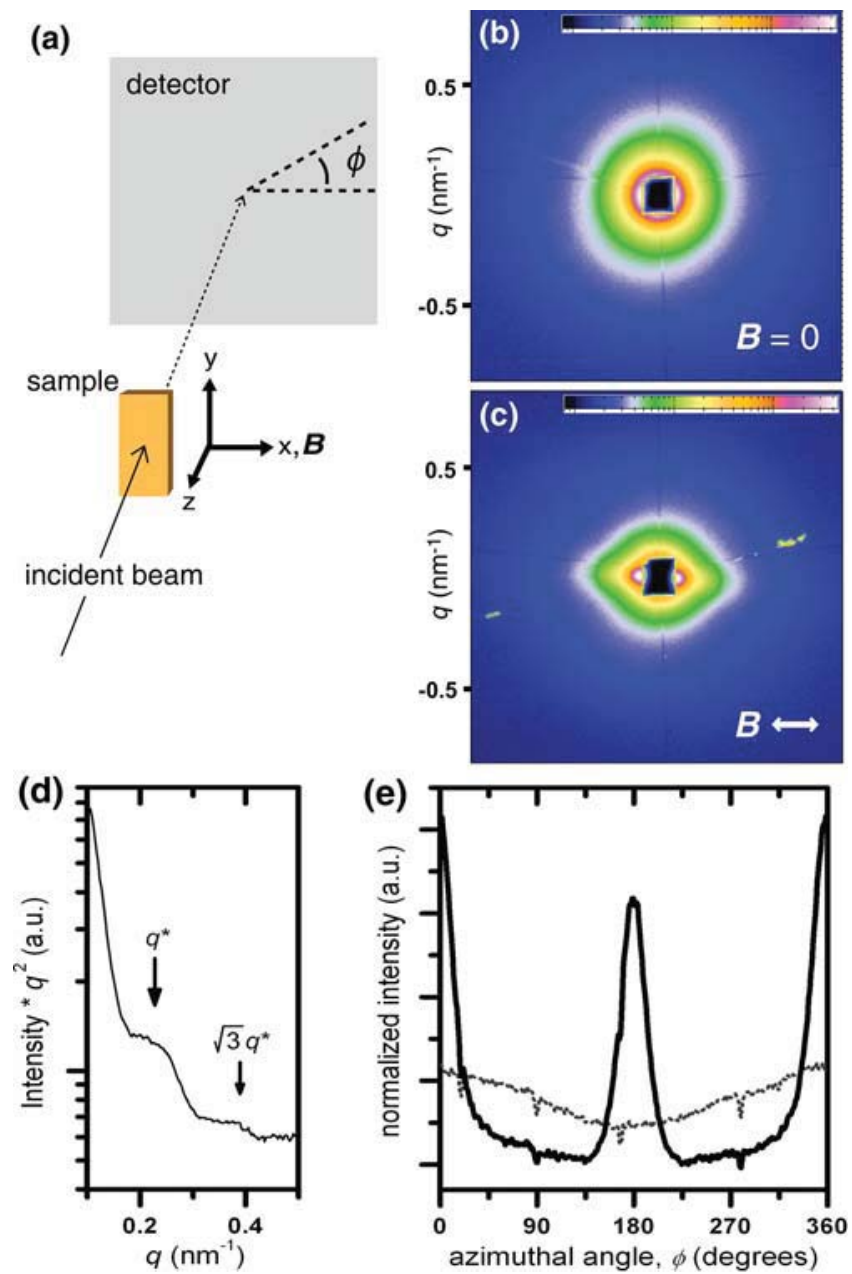

Figure 3. (a) Schematic diagram of the SAXS experiment. (b, c) Transmission SAXS patterns for BCP/NP composite films cast on mica substrates: control sample with no applied magnetic field (b) and sample with $\boldsymbol{B}$ applied as indicated (c). (d) Azimuthal average of data from the control sample, where the Bragg peaks related to the hexagonal BCP cylinder packing $\left(q^{*}\right.$ and $\left.\sqrt{ } 3 q^{*}\right)$ are indicated with arrows. (e) Azimuthal intensity scans at $q^{*}=0.23 \mathrm{~nm}^{-1}$ for the control sample (dashed gray line) and for the magnetically aligned sample (solid black line).

incorporation of defects in the cylinder array, a fact that precludes the possibility of a high degree of translational ordering of the cylinders. Nonetheless, the particles appear to effectively template the orientation of nearby cylinders. ${ }^{12,13}$ This point is driven home by the observations of regions of poor alignment (with respect to the preferred alignment direction dictated by $\boldsymbol{B}$ ) that are often observed in the TEM images as well. Crucially, those areas of misaligned cylinders are typically associated with nearby particles that are misaligned as well. In Figure $2 \mathrm{~d}$, the preferred alignment is with particles and cylinders normal to the image (right side of the image). However, two misaligned particles, lying in the plane of the section, appear to have induced misalignment in the cylinders above them (left side of the image).

In general, the TEM images suggest that the particles are effective at templating the orientation of nearby cylinders and that the magnetic alignment of the nanoparticles works to some degree, but certainly not perfectly. However, quantifying the effect requires a complementary technique with much greater statistical averaging. To this end, small-angle X-ray scattering (SAXS) was performed in transmission geometry on samples cast on thin mica substrates, the results of which are shown in Figure 3. A control sample cast and annealed with zero applied magnetic 
whereas the magnetically aligned sample shows anisotropic scattering (Figure 3c). The difference in electron density between hematite and either block of the copolymer is much larger than that between PS and PVP, so the scattering intensity at low $q$ is dominated by scattering from the particles. Attempts to isolate the contribution from the BCP structure by subtraction of the contribution to the scattering signal from only the particles proved unsuccessful. An oriented BCP array is expected to yield a mirror-image pair of spots or crescents of intensity, ${ }^{16}$ as opposed to the isotropic ring of intensity observed for unoriented samples. In Figure 3c, the lemon-shaped scattering pattern arises from the form factor of the (partially oriented) particles, ${ }^{22}$ dwarfing the signal from the BCP. However, as shown in Figure 3d, upon azimuthal integration of the scattering patterns, weak Bragg peaks associated with the BCP cylinders can nonetheless be observed at $q^{*}=0.23 \mathrm{~nm}^{-1}$ and $q \approx \sqrt{ } 3 q^{*}$. The corresponding repeat spacing, $d_{10}=27 \mathrm{~nm}$, correlates well with that observed in the TEM images. An intensity scan (as a function of azimuthal angle, $\phi$ ) taken at $q=q^{*}$, as shown in Figure 3e, thus can be used to estimate the degree of alignment of the BCP cylinders. Neglecting cylinders that do not lie parallel to the film plane (which seems reasonable given the TEM observations), the order parameter $\left\langle S_{2}\right\rangle$, which quantifies the degree of orientation of the cylinders along the preferred $(y)$ direction, can be calculated from the data in Figure 3e, viz.

$$
\left\langle S_{2}\right\rangle=\frac{3\left\langle\cos ^{2} \phi\right\rangle-1}{2}
$$

where

$$
\left\langle\cos ^{2} \phi\right\rangle=\frac{\int_{0}^{2 \pi} I(\phi) \cos ^{2} \phi|\sin \phi| \mathrm{d} \phi}{\int_{0}^{2 \pi} I(\phi)|\sin \phi| \mathrm{d} \phi}
$$

$\phi$ is defined with respect to the applied magnetic field direction ( $x$, as shown in Figure $3 a$ ) and $I(\phi)$ is the scattered intensity as a function of $\phi$. A perfectly aligned sample would yield $\left\langle S_{2}\right\rangle=1.0$, while a perfectly isotropic sample would yield $\left\langle S_{2}\right\rangle=0.0$. Calculations based on the data in Figure 3 yield values of $\left\langle S_{2}\right\rangle=0.00$ $( \pm 0.01)$ for the control sample and $\left\langle S_{2}\right\rangle=0.07( \pm 0.01)$ for the sample cast and annealed under the influence of the magnetic field. It must be noted that this calculation is sensitive to any background scattering, and as we have thus far been unsuccessful in separating scattering signals from the particles from that of the $\mathrm{BCP}$, it is unlikely that this value $\left(\left\langle S_{2}\right\rangle=0.07\right)$ is definitive.

In any case, it is clear that the overall degree of orientation produced by the magnetic field treatment in the composite is only rather modest. However, we believe the method to be general and, upon further improvements, capable of producing relatively well-aligned composites. The observation that misaligned cylinders correlate with misaligned particles suggests that improved particle alignment is of paramount importance. The fact that the hematite particles used in these experiments align with the particle long axis perpendicular to the applied magnetic field is particularly disadvantageous. The use of particles that orient parallel to an applied magnetic field should yield dramatically improved results and should allow for the creation of aligned composites in bulk form. As it is, during the film casting procedure, once the drying $\mathrm{BCP} / \mathrm{NP}$ solution reaches a certain viscosity, hydrodynamic forces on the particles will dominate over the aligning torque produced by magnetic forces. Hematite particles can in principle be transformed to maghemite $\left(\gamma-\mathrm{Fe}_{2} \mathrm{O}_{3}\right)$, in which case the particles would align parallel to the applied field. ${ }^{28}$ However, the larger remanent moment in maghemite particles creates problems in preventing particle aggregation due to increased particle-particle attraction. Such particles also tend to migrate to areas of highest magnetic field strength due to their high magnetic susceptibility (in the case of a nonhomogeneous external magnetic field), creating additional difficulties in the preparation of well-dispersed composites. In principle, these challenges can be overcome.

In summary, in a composite of spindle-type magnetic nanoparticles and cylinder-forming block copolymer, the nanoneedles have been shown to effectively template the orientation of nearby $\mathrm{BCP}$ cylinders. By application of a uniaxial magnetic field during composite casting from solution, the particles can be partially aligned, imparting an overall alignment to the final composite. At present, the alignment that has been achieved is modest at best. However, future improvements in the alignment should be possible, and in principle, any anisotropic particle with sufficient magnetic susceptibility may be used. ${ }^{29}$ Therefore, these results suggest a simple and general processing route to complex multifunctional composite materials with unique combinations of anisotropic (and orthogonally engineered) mechanical, magnetic, optical, electrical, or other properties.

Acknowledgment. Financial support was provided by the Adolphe Merkle Foundation, by the European Project "Nanomodel" within the frame of the FP7 program, and by COST D43. Alexander Hexemer and Cheng Wang at the Advanced Light Source (ALS), Lawrence Berkeley National Laboratories, provided technical assistance. The ALS is supported by the Director, Office of Science, Office of Basic Energy Sciences, of the U.S. Department of Energy under Contract DE-AC02-05CH11231. Raffaele Mezzenga and Ronald L. Jones are gratefully acknowledged for useful discussions.

Supporting Information Available: Detailed experimental procedures and analysis of particle size distributions. This material is available free of charge via the Internet at http:// pubs.acs.org.

\section{References and Notes}

(1) Vaia, R. A.; Maguire, J. F. Chem. Mater. 2007, 19, 2736-2751.

(2) Hamley, I. W. The Physics of Block Copolymers; Oxford University Press: Oxford, UK, 1998.

(3) Bockstaller, M. R.; Mickiewicz, R. A.; Thomas, E. L. Adv. Mater. 2005, 17, 1331-1349.

(4) Xu, C.; Ohno, K.; Ladmiral, V.; Composto, R. J. Polymer 2008, 49, 3568-3577.

(5) Sakurai, S. Polymer 2008, 49, 2781-2796.

(6) Hamley, I. W. J. Phys: Condens. Matter 2001, 13, R643-R671.

(7) Amundson, K.; Helfand, E.; Davis, D. D.; Quan, X.; Patel, S. S.; Smith, S. D. Macromolecules 1991, 24, 6546-6548.

(8) Russell, T. P.; Coulon, G.; Deline, V. R.; Miller, D. C. Macromolecules 1989, 22, 4600-4606.

(9) Yokoyama, H.; Mates, T. E.; Kramer, E. J. Macromolecules 2000, 33, 1888-1898.

(10) Sundrani, D.; Darling, S. B.; Sibener, S. J. Nano Lett. 2004, 4, 273 276.

(11) Hammond, M. R.; Kramer, E. J. Macromolecules 2006, 39, 15381544.

(12) Laicer, C. S. T.; Chastek, T. Q.; Lodge, T. P.; Taton, T. A. Macromolecules 2005, 38, 9749-9756.

(13) Laicer, C. S. T.; Mrozek, R. A.; Taton, T. A. Polymer 2007, 48, 1316-1328.

(14) Ferri, D.; Wolff, D.; Springer, J.; Francescangeli, O.; Laus, M.; Angeloni, A. S.; Galli, G.; Chiellini, E. J. Polym. Sci., Part B: Polym. Phys. 1998, 36, 21-29.

(15) Hamley, I. W.; Castelletto, V.; Lu, Z. B.; Imrie, C. T.; Itoh, T.; Al-Hussein, M. Macromolecules 2004, 37, 4798-4807.

(16) Tao, Y.; Zohar, H.; Olsen, B. D.; Segalman, R. A. Nano Lett. 2007, 7, 2742-2746

(17) Grigorova, T.; Pispas, S.; Hadjichristidis, N.; Thurn-Albrecht, T. Macromolecules 2005, 38, 7430-7433.

(18) Ramos, L.; Fabre, P.; Fruchter, L. Eur. Phys. J. B 1999, 8, 67-72.

(19) Béneut, K.; Constantin, D.; Davidson, P.; Dessombz, A.; Chanéac, C. Langmuir 2008, 24, 8205-8209. 
(20) PS-P2VP was purchased from PolymerSource. Molecular weights of PS and P2VP were 32000 and $12500 \mathrm{~g} / \mathrm{mol}$, respectively, and $\mathrm{PDI}=1.06$

(21) Ocaña, M.; Morales, M. P.; Serna, C. J. J. Colloid Interface Sci. 1999, 212, 317-323.

(22) Reufer, M.; Dietsch, H.; Gasser, U.; Hirt, A. M.; Menzel, A.; Schurtenberger, P. J. Phys. Chem. B 2010, 114, 4763-4769.

(23) A pair of neodymium (NIB) permanent magnets (purchased from www.supermagnete.ch) with a gap of $\sim 5 \mathrm{~mm}$ resulted in a magnetic field of $\sim 900 \mathrm{mT}$.

(24) Kim, B. J.; Chiu, J. J.; Yi, G.-R.; Pine, D. J.; Kramer, E. J. $A d v$. Mater. 2005, 17, 2618-2622.

(25) Kim, B. J.; Given-Beck, S.; Bang, J.; Hawker, C. J.; Kramer, E. J. Macromolecules 2007, 40, 1796-1798.

(26) Kim, S.; Diana, F. S.; Petroff, P. M.; Kramer, E. J. J. Polym. Sci., Part B: Polym. Phys. 2006, 44, 3227-3233.
(27) Further in evidence for the formation of a PS-P2VP brush layer is the following. The as-synthesized particles can be dispersed in THF upon sonication but will slowly sediment. Once a small amount of BCP is added, however, the particles form stable dispersions in THF.

(28) Ngo, A. T.; Pileni, M. P. J. Appl. Phys. 2002, 92, 4649-4652.

(29) Magnetic orientation of montmorillonite (MMT)/epoxy composites has been reported, taking advantage of the magnetic susceptibility of the aluminosilicate layers in some MMTs. Thus, templating a unique orientation of lamellar BCP domains should be possible via the incorporation of a low concentration of appropriately functionalized, exfoliated MMT and magnetic alignment, in analogy to what we have demonstrated here with nanorods and BCP cylinders. Koerner, H.; Hampton, E.; Dean, D.; Turgut, Z.; Drummer, L.; Mirau, P.; Vaia, R. Chem. Mater. 2005, 17, 19901996. 